\title{
Neurobiological and connectivity changes after sports-related concussion
}

\author{
Mark Krongold, Nelson Chow \\ Faculty Reviewer: G. Bryan Young, MD, FRCPC (Department of Clinical Neurological Sciences)
}

\section{ABSTRACT}

Traumatic brain injury (TBI) is one of the leading causes of morbidity and mortality worldwide. Sports-related TBI is a subset that encompasses cerebral concussion and chronic traumatic encephalopathy (CTE), the latter of which is a long-term neurodegenerative sequela of repeated mild TBI that affects behaviour, cognition, motor control, and memory. On a cellular level, TBI can result in diffuse axonal injury (DAI). This injury causes axonal transport dysfunction, leading to accumulation of tau and amyloid beta deposits in the brain. Damage occurs in neuronal tracts of both local and distant brain regions. DAI disrupts brain network function, which correlates with decreased cognitive function, by impairing the default mode network's (DMN) normal ability to deactivate during cognitive tasks. The salience network (SN) can be affected by DAI as well, which ultimately also impairs deactivation of the DMN. These changes coincide with the clinical manifestations of concussions and CTE. Both concussions and CTE are currently clinical diagnoses, as no diagnostic lab tests exist to delineate these conditions. As with many brain disorders of traumatic origin, there is no specific medical treatment for these conditions, though concussion is managed through physical and cognitive rest. The most important consideration for all TBIs, however, is prevention.

\section{INTRODUCTION}

The human brain is an exquisitely complex biological structure and is vital to the processes of life. Brain injuries can result in some of the most serious conditions involving deficits in voluntary movements, sensation, comprehension, memory, behaviour, consciousness, and more. The incidence of traumatic brain injury (TBI) has been estimated at 1.5 million a year or approximately 528 cases per 100,000, based on US data from $2003 .{ }^{1}$

One of the most common ways in which brain injury occurs is cerebral concussions from sports-related injuries. ${ }^{2}$ Concussions are an acute diffuse brain injury characterized by immediate altered brain function, with or without the loss of consciousness. Over time, repetitive mild TBI can give rise to degenerative conditions including chronic traumatic encephalopathy (CTE), which is characterized by cognitive and behavioural impairments. ${ }^{2}$

\section{CLINICAL FEATURES AND MANAGEMENT}

Accurate recognition and appropriate management of acute TBI is critical in preventing further injury, especially in the context of concussion. Despite our knowledge of concussion pathology from structural imaging studies, no definitive diagnostic lab test or imaging currently exists for concussions; it remains a clinical diagnosis based on assessment of signs and symptoms. ${ }^{3}$ While diagnostic imaging appears normal for mild concussions, more serious injuries such as skull fractures and intracranial hemorrhages can still be assessed with CT or MRI. ${ }^{3}$

Guidelines for assessing concussion generally categorize the signs and symptoms into 3 or 4 major categories: physical, cognitive, emotional/behavioural, and sleep-related. ${ }^{3-5}$ These can present up to a week after injury, thus utilizing scoring symptoms with checklists, assessing balance, and testing neuropsychological function provides much greater sensitivity for diagnosis. ${ }^{\circ}$

There are also complications that can follow concussion, including second impact syndrome, post-concussion syndrome, and CTE. Second impact syndrome occurs when a person suffering a previous concussion receives another TBI during the recovery period which can lead to diffuse cerebral swelling and mortality. ${ }^{7}$ In other cases, concussive symptoms may not resolve within the usual period, a condition called post-concussive syndrome., ${ }^{2,4}$ Recognizing CTE is more difficult, because while it also presents with behavioural, cognitive, and motor dysfunction, its insidious nature can result in a delay of many years in symptom presentation. ${ }^{2}$

Diagnosing CTE is based on cognitive and/or behavioural impairment, cerebellar dysfunction, and pyramidal or extrapyramidal disease that can be distinguished from other causes such as Parkinson's disease, Alzheimer's disease, and cerebrovascular damage. ${ }^{2}$ A definitive diagnosis requires confirmation of tau deposits, which is only possible via autopsy. ${ }^{2}$

Due to the increased susceptibility to damage after TBI, the management of concussion is focused on preventing further injury which is governed by 'return to play' criteria in sports. ${ }^{3}$ This involves immediate cessation of play and resting both physically and cognitively until pre-injury function is restored, which typically occurs within two weeks. ${ }^{3}$ Prolonged recovery periods and signs of chronic symptoms may require further management. Although options are limited to treatment of symptoms, some research suggests aerobic exercise may be therapeutic. ${ }^{8}$

There exists no specific treatment for CTE, emphasizing the importance of prevention. Proper management of acute TBI and minimizing exacerbations reduces the chances of developing CTE. ${ }^{2,9}$

\section{TRAUMATIC BRAIN INJURY PATHOLOGY}

Brain injury can be caused by focal or diffuse phenomena. Focal damage occurs when an object strikes the head causing contusion, skull fracture, or intracerebral hemorrhage, leading to direct 
cortical or subcortical neuronal loss. ${ }^{10}$ Diffuse damage largely arises from acceleration/deceleration forces acting on the brain causing shearing (stretching and tearing) damage to blood vessels as well as long distance neuronal connections, which is known as diffuse axonal injury (DAI). ${ }^{11,12}$ At the cellular level DAI is marked by abnormal metabolism, calcium influx, microtubule disassembly and cytoskeletal dysfunction causing impaired axonal protein transport, abnormal protein accumulation, neuronal circuit disruption and axonal degeneration. ${ }^{13,14}$

Most damage to the brain after TBI is not due to the initial insult but from the progressive deterioration secondary to pathological processes that are triggered by the insult. ${ }^{15}$ This deterioration is a long-term process that can take place over hours to years and is not limited to the focal injury site. ${ }^{16}$ Secondary damage mechanisms include cytotoxicity, ischemia, increased intracranial pressure from edema, infection, inflammation, and neurodegeneration. . $^{15,17,18}$

Neurodegeneration after TBI is marked by the accumulation of amyloid beta and neurofibrillary tangles. ${ }^{9,18,19}$ After neuronal injury has occurred, the expression of amyloid precursor protein (APP) is increased. ${ }^{20}$ The accumulated amyloid beta inside of axons is eventually expulsed into surrounding tissue, leading to plaque formation around the damaged axons. ${ }^{21,22}$ Furthermore, it has been hypothesized that disassembly of microtubules and impaired axonal transport can lead to tau, a protein that binds to microtubules, and neurofilament aggregation, resulting in tangles. ${ }^{23}$ Spread of these abnormal proteins over time away from the focal site has been associated with inflammation, ${ }^{24}$ which is caused by the activation of microglia after TBI. ${ }^{13,25,26}$ However, it is currently unknown if the inflammation precedes neurodegeneration or if it is an attempted self-repair response by the brain. ${ }^{27}$

CTE is a progressive neurodegeneration that develops due to previously damaged areas of the brain being more susceptible to repeated trauma. ${ }^{28}$ Damaged neurons undergo membrane disruption causing ion dysregulation and release of excitatory neurotransmitters. ${ }^{28,29}$ The surviving neurons respond by repairing the damaged areas, which leads to depletion of energy stores and a worsened response to repeated injury that amplifies axonal damage. ${ }^{18}$ The pathological mechanisms described above, such as amyloid beta and tau pathology, have been shown to occur in CTE and lead to cognitive decline in athletes over many years. ${ }^{9,30,31}$

\section{CHANGES IN BRAIN CONNECTIVITY}

Dysfunction in networks in the brain are thought to be a major component of many brain disorders including TBI. ${ }^{32}$ DAI is believed to be more important than focal injury in predicting clinical outcomes and cognitive impairment, as the cognitive impairments seen in TBI are due to dysfunction of brain networks. ${ }^{33-35}$ Traditional imaging techniques are not sensitive enough to find nonhemorrhagic injury such as DAI and its effect on neuronal network atrophy. ${ }^{36}$ More recently, new imaging methods such as diffusion tensor MRI and functional MRI, which can create images of structural and functional brain connectivity and help quantify the degree of white matter damage, have been used to study the effects of TBI on neuro- nal networks. ${ }^{36,37}$ Finding disruptions in specific networks can help determine impairment patterns and correlate the damage to cognitive function, ${ }^{33,38,39}$ as well as functional outcomes. ${ }^{40}$

Studies of network dysfunction after TBI have found complex and sometimes contradictory patterns. Of particular interest are studies on the default mode network (DMN), which is a network that is active when subjects are at rest and not performing cognitive tasks. ${ }^{17,41}$ Networks can be affected in 3 ways: disconnection, abnormal activity, and coordination dysfunction. Researchers studying the DMN have shown that increasing damage to the connections between DMN regions correlates with attention impairment and decreased functional connectivity. ${ }^{34}$ The DMN is known to deactivate in healthy controls when subjects perform tasks that require increased attention, but in subjects with TBI there is a failure of the network to deactivate which can lead to poor cognitive function. ${ }^{42,43}$ Accordingly, coordination between the DMN and another network called the salience network (SN) has been found to be affected after TBI. The SN is responsible for helping to deactivate the DMN in healthy subjects, but TBI that damages parts of the SN results in a failure of DMN deactivation. ${ }^{42}$

There have also been a number of studies of functional connectivity changes in the brain that demonstrate increased and decreased activity in different networks. ${ }^{17}$ The damage that occurs in the brain after TBI is complex: injury to a single neuronal tract can have effects both locally and in distant brain regions to which that tract connects. As this is difficult to investigate in humans using current technology, researchers have begun to study TBI lesions in specific neuronal tracts in animal models. ${ }^{17}$ At this point, however, diffusion MRI is not used clinically to diagnose or treat TBI. ${ }^{17}$ Once these tools are developed, network level descriptions of structural and functional damage and their associations with cognitive impairment will be able to be used for long-term outcome prediction. Furthermore, drugs or other methods of brain network stimulation/inhibition, such as transcranial magnetic stimulation, might then be used to treat network dysfunction. ${ }^{17}$

\section{CONCLUSION}

Traumatic brain injuries pose a significant risk to brain function, and due to their incurable nature require consideration of preventative measures and management to minimize further damage in the context of sports-related injuries. Moreover, due to the increased likelihood of repeated mild TBI, sports-related TBI can lead to CTE through the accumulation of tau and amyloid deposits, as well as network dysfunction.

Further development of diagnostic techniques such as biomarkers and higher resolution structural imaging will be needed to enable accurate diagnosis of concussion and CTE, and may allow for monitoring of the disease course during treatment. 


\section{REFERENCES}

1. Rutland-Brown W, Langlois JA, Thomas KE, et al. Incidence of traumatic brain injury in the United States, 2003. J Head Trauma Rehabil. 2006;21(6):544-8.

2. Jordan BD. The clinical spectrum of sport-related traumatic brain injury. Nat Rev Neurol. 2013 Apr;9(4):222-30.

3. Graham R, Rivara FP, Ford MA. Concussion Recognition, Diagnosis, and Acute Management. In: Sports-Related Concussions in Youth: Improving the Science, Changing the Culture. Washington (DC): National Academies Press (US); 2014.

4. Willer B, Leddy JJ. Management of concussion and post-concussion syndrome. Curr Treat Options Neurol. 2006 Sep;8(5):415-26.

5. Marshall S, Bayley M, McCullagh S, et al. Clinical practice guidelines for mild traumatic brain injury and persistent symptoms. Can Fam Physician. 2012 Mar;58(3):257-67, e128-40.

6. Broglio SP, Macciocchi SN, Ferrara MS. Sensitivity of the concussion assessment battery. Neurosurgery. 2007 Jun;60(6):1050-7; discussion 1057-8.

7. Bey T, Ostick B. Second impact syndrome. West J Emerg Med. 2009 Feb;10(1):6-10.

8. Graham R, Rivara F, Ford M. Treatment and Management of Prolonged Symptoms and Post-Concussion Syndrome. In: Sports-Related Concussions in Youth: Improving the Science, Changing the Culture. Washington (DC): National Academies Press (US); 2014.

9. McKee AC, Cantu RC, Nowinski CJ, et al. Chronic traumatic encephalopathy in athletes: progressive tauopathy after repetitive head injury. $\mathrm{J}$ Neuropathol Exp Neurol. 2009 Jul;68(7):709-35.

10. Graham DI, McIntosh TK, Maxwell WL, et al. Recent advances in neurotrauma. J Neuropathol Exp Neurol. 2000 Aug;59(8):641-51.

11. Smith DH, Meaney DF, Shull WH. Diffuse axonal injury in head trauma. J Head Trauma Rehabil. 2003;18(4):307-16.

12. Werner C, Engelhard K. Pathophysiology of traumatic brain injury. Br J Anaesth. 2007 Jul;99(1):4-9.

13. Johnson VE, Stewart W, Smith DH. Axonal pathology in traumatic brain injury. Exp Neurol. 2013 Aug;246:35-43.

14. Chen X-H, Siman R, Iwata A, et al. Long-term accumulation of amyloid-beta, beta-secretase, presenilin-1, and caspase-3 in damaged axons following brain trauma. Am J Pathol. 2004 Aug;165(2):357-71.

15. Chen Y, Garcia GE, Huang W, et al. The Involvement of Secondary Neuronal Damage in the Development of Neuropsychiatric Disorders Following Brain Insults. Front Neurol. 2014 Mar 11;5.

16. Chen Y. Organophosphate-induced brain damage: Mechanisms, neuropsychiatric and neurological consequences, and potential therapeutic strategies. Neurotoxicology. 2012 Jun;33(3):391-400.

17. Sharp DJ, Scott G, Leech R. Network dysfunction after traumatic brain injury. Nat Rev Neurol. 2014 Feb 11;10(3):156-66.

18. Blennow K, Hardy J, Zetterberg H. The Neuropathology and Neurobiology of Traumatic Brain Injury. Neuron. 2012 Dec;76(5):886-99.

19. Smith DH, Johnson VE, Stewart W. Chronic neuropathologies of single and repetitive TBI: substrates of dementia? Nat Rev Neurol. 2013 Apr;9(4):211-21.

20. Chen Y, Tang BL. The amyloid precursor protein and postnatal neurogenesis/neuroregeneration. Biochem Biophys Res Commun. 2006 Mar 3;341(1):1-5.

21. Chen X-H, Johnson VE, Uryu K, et al. A lack of amyloid beta plaques despite persistent accumulation of amyloid beta in axons of long-term survivors of traumatic brain injury. Brain Pathol. 2009 Apr;19(2):214-23.

22. Smith DH, Chen X-H, Iwata A, et al. Amyloid beta accumulation in axons after traumatic brain injury in humans. J Neurosurg. 2003 May;98(5):1072-7.

23. Mandelkow E-M, Mandelkow E. Biochemistry and cell biology of tau protein in neurofibrillary degeneration. Cold Spring Harb Perspect Med. $2012 \mathrm{Jul} ; 2$ (7):a006247.

24. Holmin S, Mathiesen T. Long-term intracerebral inflammatory response after experimental focal brain injury in rat. Neuroreport. 1999 Jun 23;10(9):1889-91.

25. Ramlackhansingh AF, Brooks DJ, Greenwood RJ, et al. Inflammation after trauma: microglial activation and traumatic brain injury. Ann Neurol. 2011 Sep;70(3):374-83.

26. Shitaka Y, Tran HT, Bennett RE, et al. Repetitive closed-skull traumatic brain injury in mice causes persistent multifocal axonal injury and microglial reactivity. J Neuropathol Exp Neurol. 2011 Jul;70(7):551-67.

27. Loane DJ, Byrnes KR. Role of microglia in neurotrauma. Neurotherapeutics. 2010 Oct;7(4):366-77.

28. Barkhoudarian G, Hovda DA, Giza CC. The molecular pathophysiology of concussive brain injury. Clin Sports Med. 2011 Jan;30(1):33-48, vii - iii.

29. Spain A, Daumas S, Lifshitz J, et al. Mild fluid percussion injury in mice produces evolving selective axonal pathology and cognitive deficits relevant to human brain injury. J Neurotrauma. 2010 Aug;27(8):1429-38.

30. Omalu BI, DeKosky ST, Minster RL, et al. Chronic traumatic encephalopathy in a National Football League player. Neurosurgery. 2005 Jul;57(1):128-34; discussion 128-34.

31. Yi J, Padalino DJ, Chin LS, et al. Chronic traumatic encephalopathy. Curr Sports Med Rep. 2013;12(1):28-32.

32. Zhang D, Raichle ME. Disease and the brain's dark energy. Nat Rev Neurol. 2010 Jan;6(1):15-28.

33. Kinnunen KM, Greenwood R, Powell JH, et al. White matter damage and cognitive impairment after traumatic brain injury. Brain. $2011 \mathrm{Feb} ; 134(\mathrm{Pt}$ 2):449-63.

34. Bonnelle V, Leech R, Kinnunen KM, et al. Default mode network connectivity predicts sustained attention deficits after traumatic brain injury. $\mathrm{J}$ Neurosci. 2011 Sep 21;31(38):13442-51.

35. Hellyer PJ, Leech R, Ham TE, et al. Individual prediction of white matter injury following traumatic brain injury. Ann Neurol. 2013 Apr;73(4):48999.

36. Irimia A, Wang B, Aylward SR, et al. Neuroimaging of structural pathology and connectomics in traumatic brain injury: Toward personalized outcome prediction. NeuroImage Clin. 2012;1(1):1-17.

37. Mac Donald CL, Dikranian K, Bayly P, et al. Diffusion tensor imaging reliably detects experimental traumatic axonal injury and indicates approximate time of injury. J Neurosci. 2007 Oct 31;27(44):11869-76.

38. Caeyenberghs K, Leemans A, Leunissen I, et al. Altered structural networks and executive deficits in traumatic brain injury patients. Brain Struct Funct. 2014 Jan;219(1):193-209.

39. Strangman GE, O’Neil-Pirozzi TM, Supelana C, et al. Fractional anisotropy helps predicts memory rehabilitation outcome after traumatic brain injury. NeuroRehabilitation. 2012;31(3):295-310.

40. Sidaros A, Engberg AW, Sidaros K, et al. Diffusion tensor imaging during recovery from severe traumatic brain injury and relation to clinical outcome: a longitudinal study. Brain. 2008 Feb;131(Pt 2):559-72.

41. Raichle ME, MacLeod AM, Snyder AZ, et al. A default mode of brain function. Proc Natl Acad Sci U S A. 2001 Jan 16;98(2):676-82.

42. Bonnelle V, Ham TE, Leech R, et al. Salience network integrity predicts default mode network function after traumatic brain injury. Proc Natl Acad Sci U S A. 2012 Mar 20;109(12):4690-5.

43. Weissman DH, Roberts KC, Visscher KM, et al. The neural bases of momentary lapses in attention. Nat Neurosci. 2006 Jul;9(7):971-8. 